\title{
ATLAS Muon Trigger performance
}

\author{
Yohei Noguchi*, on behalf of the ATLAS Collaboration \\ Kyoto University \\ E-mail: vohei.noguchiecern.ch
}

\begin{abstract}
Events containing muons in the final state are important for many physics analyses being carried out at the Large Hadron Collider, including both Standard Model measurements and searches for new physics. The muon trigger in the ATLAS experiment consists of a hardware-based first level trigger, and a succeeding software-based trigger. In order to handle the high luminosity in Run-2, several upgrades have been implemented to reduce trigger rate, while keeping a high efficiency. These improvements have been done by coincidence of hits in the muon spectrometer and the calorimeter, optimisation of muon isolation, and better determination of transverse momentum. An overview of the muon trigger in ATLAS with the recent improvements is presented. Also performance of the muon trigger in Run-2 and an outlook for upgrades planned for Run-3 are presented.
\end{abstract}

XXIX International Symposium on Lepton Photon Interactions at High Energies - LeptonPhoton2019 August 5-10, 2019

Toronto, Canada

${ }^{*}$ Speaker. 


\section{Introduction}

The muon trigger in the ATLAS experiment covers phase space of muons with a wide range of transverse momentum $\left(p_{\mathrm{T}}\right)$ and pseudo-rapidity $(\eta)$. This allows us to study many physics processes from production of Higgs bosons to physics including $B$-hadrons.

The ATLAS detector has two components for reconstruction of muons: Muon Spectrometers with a toroid magnet system of 1-1.5 T and Inner Detectors (ID) with a $2 \mathrm{~T}$ solenoid magnet [四]. The Level-1 (L1) muon trigger employs Thin Gap Chambers (TGCs) and Resistive Plate Chambers (RPCs) to select events based on coarse $p_{\mathrm{T}}$ determination. The L1 trigger also defines Regions of Interest (RoIs) which are regions of the detector in $\eta$ and $\phi$ considered by the High Level Trigger. To achieve the very fast selection (40 MHz input rate, $2.5 \mu \mathrm{sec}$ latency), trigger logic is implemented on dedicated hardware (FPGAs). The muon High Level Trigger (HLT) uses dedicated software to reconstruct muons in the regions defined by L1 using information from precision trackers, Monitored Drift Tubes (MDTs), Cathode Strip Chambers (CSCs) and ID. The HLT is separated into two parts: fast and precise reconstruction algorithms. The former executes simple algorithms and provides fast selection, and the latter considers the full detector information using algorithms similar to offline reconstruction. Besides the precise $p_{\mathrm{T}}$ measurement, isolation criteria can also be used to reject non-prompt muons. The lowest threshold of single-muon trigger with isolation is $26 \mathrm{GeV}$. Triggers with lower $p_{\mathrm{T}}$ thresholds are also available, suppressing their rates by requiring multiple muons from the decays of $B$-hadrons.

\section{Improvements during Run-2}

Some upgrades in L1 and the HLT were introduced during Run-2 to keep the trigger rate acceptable while maintaining the efficiency as high as possible. As shown in Figures $\square$ and 】, the L1 muon trigger output is dominated by charged particles that are not muons but secondary particles from the scattering of the protons at the beam pipe and those protons are from low energy scatterings with small angles at the interaction point ("fake triggers/muons"). The fake triggers can

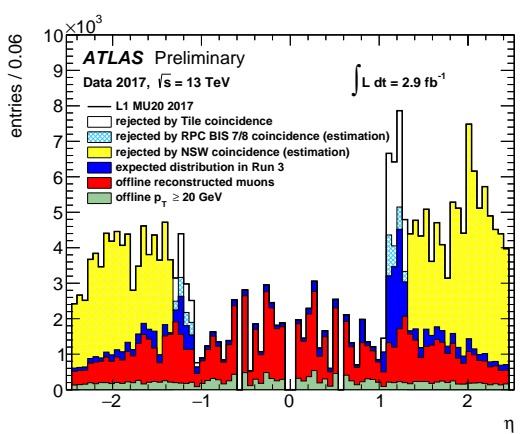

Figure 1: The $\eta$ distribution of the L1 muon RoIs with a $20 \mathrm{GeV}$ threshold [[]]. The histogram for the total entries shows the observed distribution in Run-2. The histograms with colors show the components as explained in the figure.

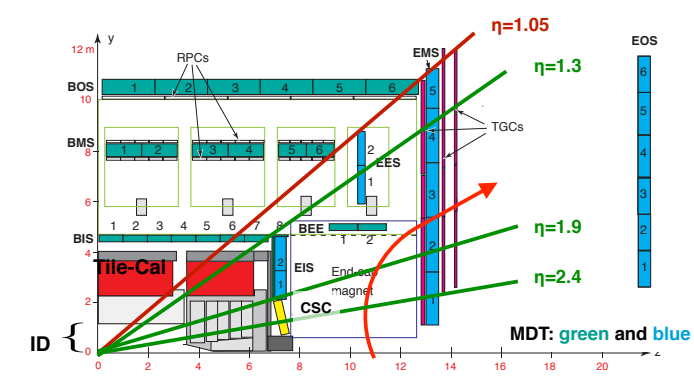

Figure 2: A quarter of the cross-section of the ATLAS Muon Spectrometer [B]]. The four components, RPC, TGC, MDT and CSC are shown in white, pink, green and blue, and yellow areas as described in the figure. The red arrow shows a trajectory of a low- $p_{\mathrm{T}}$ charged particle from the beam pipe. 


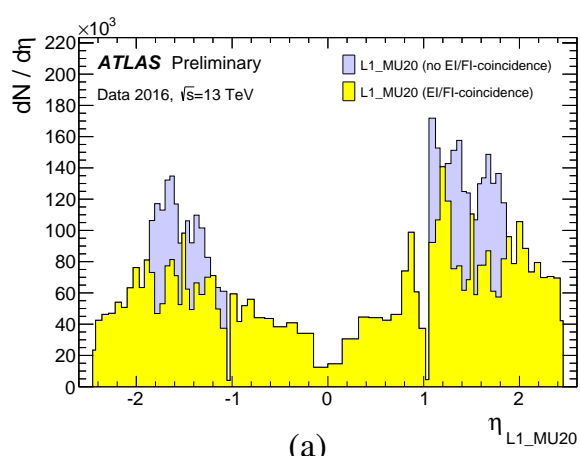

(a)

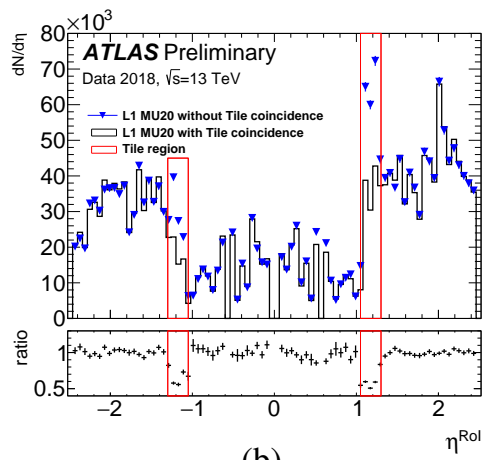

(b)

Figure 3: The $\eta$ distributions of the $\mathrm{L} 1$ muon RoIs with $20 \mathrm{GeV}$ threshold. The distribution is shown with and without the coincidence with the TGC chambers in the inner-most layer (a) and the Tile-Calorimeter (b) [ם, 四].

effectively be removed by requiring coincidence between detectors installed inside and outside of the magnet system. To suppress the fake L1 triggers in $1.05<|\eta|<1.9$, coincidence of hits in TGCs in the inner most layer and the outer layer is required. This allows us to reduce rate of the L1 trigger with a threshold of $20 \mathrm{GeV}$ by $\sim 20 \%$ as shown in Figure B(a). Coincidence between the energy detection in the Tile-Calorimeter and hits in TGCs in the outer layer is also required to suppress the fake triggers in $1.05<|\eta|<1.3$ (Figure B(b)). This allows us to achieve 6\% more rate reduction in the $\mathrm{L} 1$ muon trigger with the threshold of $20 \mathrm{GeV}$ in $\phi$ range uncovered by the inner most TGCs.

In 2018, the isolation criteria in the HLT are optimized to use tracks within $d z<2 \mathrm{~mm}$ from a muon instead of $6 \mathrm{~mm}$, in order to keep a high efficiency of isolated muon triggers even in the environment where multiple hard scatterings occur in the same events (Figure $⿴$ ). The CSC chambers are included in the fast $p_{\mathrm{T}}$ determination algorithm to improve the $p_{\mathrm{T}}$ resolution in the forward region $(2.0<|\eta|<2.4)$ (Figure \).

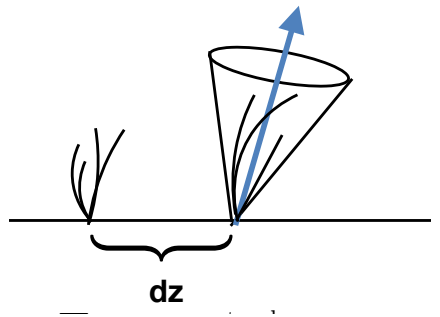

Figure 4: A schematic diagram of tracks considered in isolation criteria. The tracks around a muon within $\Delta R<$ 0.3 and $\mathrm{d} z<2 \mathrm{~mm}(6 \mathrm{~mm}$ until 2017) are used to define the isolation variable, $\sum p_{\mathrm{T}}^{\text {track }} / p_{\mathrm{T}}^{\text {muon }}<0.07$.

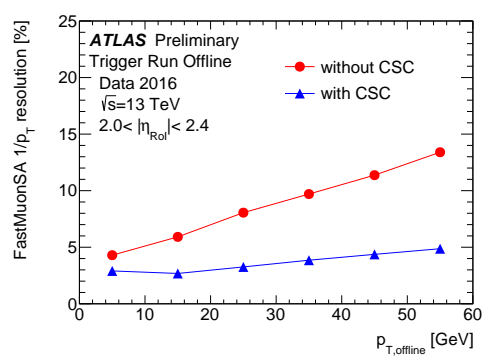

Figure 5: Improvement of $p_{\mathrm{T}}$ resolution of the fast $p_{\mathrm{T}}$ determination algorithm at HLT in $2.0<|\eta|<2.4$ by including the CSC chambers [䧃].

\section{Performance}

The measurement of the trigger efficiency is performed exploiting the "Tag \& Probe" method 


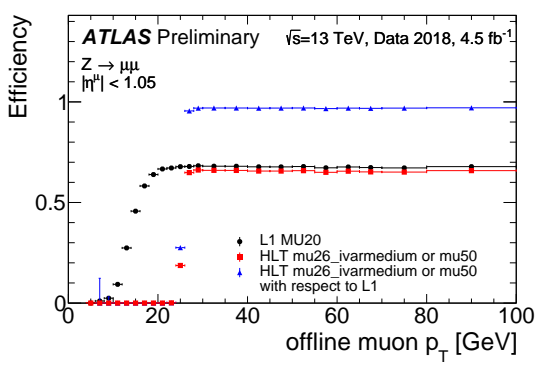

(a)

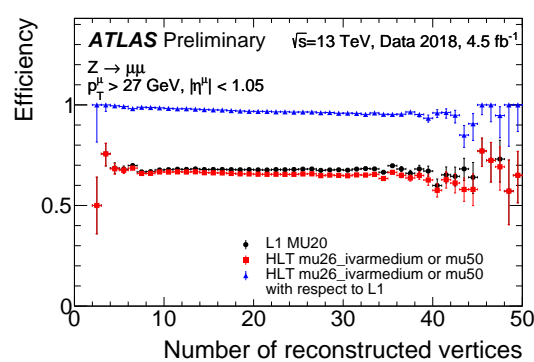

(b)

Figure 6: Efficiencies of a specific single-muon trigger for the barrel region as functions of $p_{\mathrm{T}}$ of muons (a) and the number of reconstructed vertices (b) [䧃]. The efficiency is for the OR of the trigger with $26 \mathrm{GeV} p_{\mathrm{T}}$ threshold and the isolation requirement and the trigger with $50 \mathrm{GeV} p_{\mathrm{T}}$ threshold. Efficiencies of L1, HLT and the total efficiency are shown.

using $Z \rightarrow \mu \mu$ events, which requires "tag" muon in the events to match the trigger itself and "probe" muon to be used instead for the efficiency measurement. The L1 trigger efficiency for

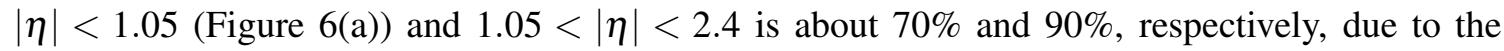
coverage of RPCs and TGCs. The HLT is almost $100 \%$ efficient with respect to L1. The observed dependence of the efficiency on the number of reconstructed vertices from $p p$ collisions is small as shown in Figure G(b).

\section{Upgrades towards Run-3}

To handle higher luminosity in Run-3 $\left(L \simeq 2.0 \times 10^{34} \mathrm{~cm}^{-2} \mathrm{~s}^{-1}\right)$, the muon chambers in the inner most layer will be replaced with higher granularity detectors called the New Small Wheels (NSW) in $1.3<|\eta|<2.7$ [[5], and new RPC chambers will be installed in $1.0<|\eta|<1.3$. Track information from these new chambers will be used in coincidence with the outer layer of TGCs to reduce the fake L1 triggers in the same way discussed in the section $\square$. To implement such an algorithm, new trigger boards are also introduced to the L1 muon trigger system. The HLT is also upgraded to make use of these new chambers. Multi-threading will be introduced to the HLT which allows us to more optimally use the available CPUs by reducing the memory usage [䧃].

\section{References}

[1] ATLAS Collaboration, 2008 JINST 3 S08003

[2] ATLAS Collaboration, https://twiki.cern.ch/twiki/bin/view/AtlasPublic/L1MuonTriggerPublicResults

[3] Joerg Wotschack, "ATLAS Muon Chamber Construction Parameters for CSC, MDT, and RPC chambers", ATL-MUON-PUB-2008-006, 2008. https://cds.cern.ch/record/1099400

[4] ATLAS Collaboration, https://twiki.cern.ch/twiki/bin/view/AtlasPublic/MuonTriggerPublicResults

[5] ATLAS Collaboration, "ATLAS New Small Wheel Technical Design Report", http://inspirehep.net/record/1615722/, 2013.

[6] John Baines et al., "ATLAS Future Framework Requirements Group Report," ATL-SOFT-PUB-2016-001, 2016. https://cds.cern.ch/record/2196967 